\title{
An analytical framework for smart manufacturing
}

\author{
Amogh Kulkarni ${ }^{1}$, Daniel Balasubramanian ${ }^{1}$, Gabor Karsai ${ }^{1}$ and Peter Denno ${ }^{2}$ \\ ${ }^{1}$ Institute for Software Integrated Systems, Vanderbilt University, Nashville, TN 37212 \\ ${ }^{2}$ National Institute of Standards and Technology, Gaithersburg, MD 20899
}

\begin{abstract}
Smart manufacturing is an emerging paradigm for the next generation of manufacturing systems. One key to the success of smart manufacturing is the ability to use the production data for defining predictive and descriptive models and their analyses. However, the development and refinement of such models is a labor- and knowledgeintensive activity that involves acquiring data, selecting and refining an analytical method and validating results. This paper presents an analytical framework that facilitates these activities by allowing ad-hoc analyses to be rapidly specified and performed. The proposed framework uses a domain-specific language to allow manufacturing experts to specify analysis models in familiar terms and includes code generators that automatically generate the lower-level artifacts needed for performing the analysis. We also describe the use of our framework with an example problem.
\end{abstract}

\section{Introduction}

Smart manufacturing systems (SMS) is an emerging paradigm for the next generation of manufacturing systems. Its goal is to enable data-driven decision making throughout manufacturing. SMS holds the potential to improve many aspects of manufacturing. For example, using real-time feedback about part quality, a SMS may adjust process parameters to optimize throughput.

Important to success in smart manufacturing is the ability to learn from data. Data informs control decisions in SMSs through two pathways yielding predictive models: where phenomena are not well-understood, data analytics (e.g. machine learning) can be applied; and where phenomena are understood, conventional analytical methods (e.g finite element analysis, operations research) are typically more effective.

Regardless of the pathway, the resultant computational model produced can be used either directly to infer control signals or "off-line" in trade studies and numerical optimizations. In both the machine learning and analytical pathways, the development and refinement of predictive models is both a labor- and knowledgeintensive activity involving the tasks of acquiring and conditioning data, selecting and refining an analytical method (e.g. experimental, physics-based etc.), and validating results. These tasks could be facilitated by a system that integrates access and visualization of production data with analytical capabilities. This paper describes the design and implementation of such a system.

There are several challenges to designing and building this type of framework. First, data may be produced in a variety of different formats, yet flexible tools to examine and organize the data for analysis are needed. Second, one must provide access to multiple analysis algorithms.
Third, the framework should allow users to combine both process concerns and operations concerns in the analysis. Fourth, the framework should facilitate continual refinement of the analytical model. Finally, the framework must present the analysis results in a way that allows insights and actionable conclusions to be drawn.

This paper presents an analytical framework to support decision making in smart manufacturing settings. The paper focuses on the aspects of the framework that allow ad-hoc analyses to be rapidly specified and performed. The framework supports input data in the MTConnect standard [1], an industry standard for describing data produced by machine tools. It stores data using InfluxDB [2], a time-series database built for realtime analytics applications. The framework also integrates various Python libraries like OpenMDAO [3] (which provides analysis algorithms), SimPy [4] (for developing discrete event simulation models of manufacturing processes), scikit-learn [5] (for developing data-driven models of manufacturing processes) and Bokeh [6] (for visualizing analysis results). Grafana [7] visualization engine is used to visualize the data logged in InfluxDB. Underpinning the framework is a domainspecific modeling language that allows users to express complex analyses using an intuitive, extensible modeling language.

The rest of this paper is structured as follows. Section 2 gives brief background information on MTConnect and model-based software engineering. Section 3 describes the application of the proposed framework to a motivating example. Related work is presented in Section 4 and section 5 gives the design and implementation of the framework. Finally, section 6 concludes the discussion and outlines the future work. 


\section{Background}

Attempts to create the network of "things" in a manufacturing setting date back to the 1970 s, but were largely unsuccessful because of immature communication infrastructure, lack of facilities for large data storage, and limitations on computing power. Today, these impediments no longer exist and we are observing a fusion of manufacturing systems and Cyber-Physical Systems (CPS) which has given rise to the field of Cyber Manufacturing [8]. "Industrial Internet-of-Things" (IIoT) provide new opportunities to improve process quality, make predictions, and perform fault-diagnosis and prognosis. In this new paradigm, communication standards play a very important role.

MTConnect is a communication standard aimed at making factory data from Computer Numerical Control (CNC) machines machine-readable and more accessible. The development of standards like MTConnect have paved the way towards cross-platform analytical applications and sensor fusion. Although empirical techniques for data acquisition can be used for most of the analytics applications, the integration of CyberPhysical Systems with modern manufacturing techniques provides more efficient means to acquire data $[9,10]$. MTConnect enables near real-time streaming of factory data which can be effectively used for the analysis of manufacturing processes.

Model-Based System Engineering (MBSE) is an effective approach for developing CPS applications as the transition from document-based to model-based methodologies continues [11]. This is especially the case as systems and processes grow more complex and require the integration and composition of multiple heterogeneous models [12]. Domain-specific modeling is a part of MBSE that deals with modeling environments to provide abstractions that cater to specific engineering disciplines. The framework described in this paper uses a Domain-Specific Modeling Language (DSML) to help small- and medium-scale manufacturers leverage the MBSE paradigm for performing manufacturing analysis.

\section{Motivating example}

The motivating example used to explain the framework is a machining process running on a MTConnect-enabled CNC workcenter. 20 copies of a milled Aluminium part were produced using end milling and face milling at various feeds and speeds. This aggregate dataset is used to produce a predictive model of the process using standard regression techniques. The regression model predicts the time required to produce a part for the aggregated value of spindle speed while producing a part.

The MTConnect data produced during the milling process under study contains the values of various process parameters such as spindle speed and line feeds during the production of the part. In addition to these process parameters, the MTConnect data contains information about the state of the CNC machine during production. Using these data streams, statistical analyses like linear regression can be performed to determine relationships such as that between values of process parameters and changes in the state of the machining tool. Such statistical models help to approximate the relationship between the process parameters and process performance metrics, where obtaining the accurate analytical relationship between the two is not possible.

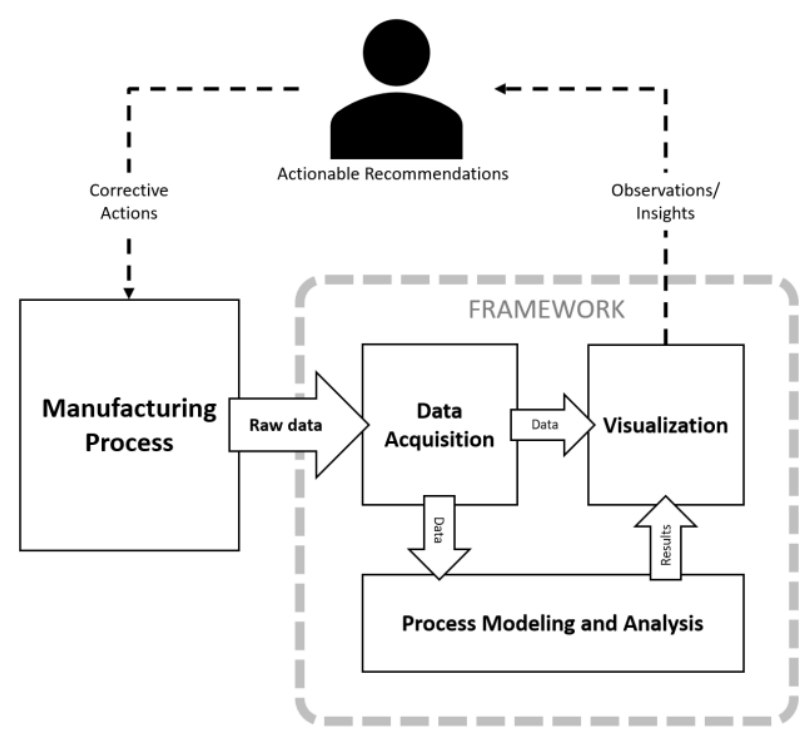

Figure 1. Overview of the framework

Figure 1 gives a high-level view of the entire workflow. The manufacturing process under question can be considered as a "black-box" that produces the raw data. This data is saved in a data acquisition module which is integrated with a domain-specific modeling environment. It can be used to develop the models of the process in question, which in turn can be used to perform the analyses. Visualization of both the input data as well as the analysis results provide the user with valuable insights about the nature of the process.

There is a vast body of literature on predictive, diagnostic, and descriptive analysis of manufacturing processes. In discrete part manufacturing, surface finish and dimensional accuracy are key to part quality. Studies predicting surface roughness have used factorial designof-experiment in turning processes [13], regression and neural-network based approaches [14], and empirical models developed from the data [15]. Predictive analysis of process efficiency includes the prediction of energy consumption (i.e. sustainability analysis) by developing empirical models using data [16] and prediction of tool wear using neural networks [17]. Studies estimating process properties such as manufacturing cycle time have been done using data mining approaches on stored data in the semiconductor manufacturing domain [18].

The typical workflow of the approaches listed above is to (1) gather the data from the process, (2) develop a data-driven model or an analytical model, and (3) use the model and acquired data to perform various analyses to gain insights about the system. The difficulty with this workflow is that it requires expertise in data acquisition techniques, efficient data storage techniques, and advanced programming skills to implement and/or use the modeling platforms and data visualization techniques. The work in this paper addresses this need and provide an 


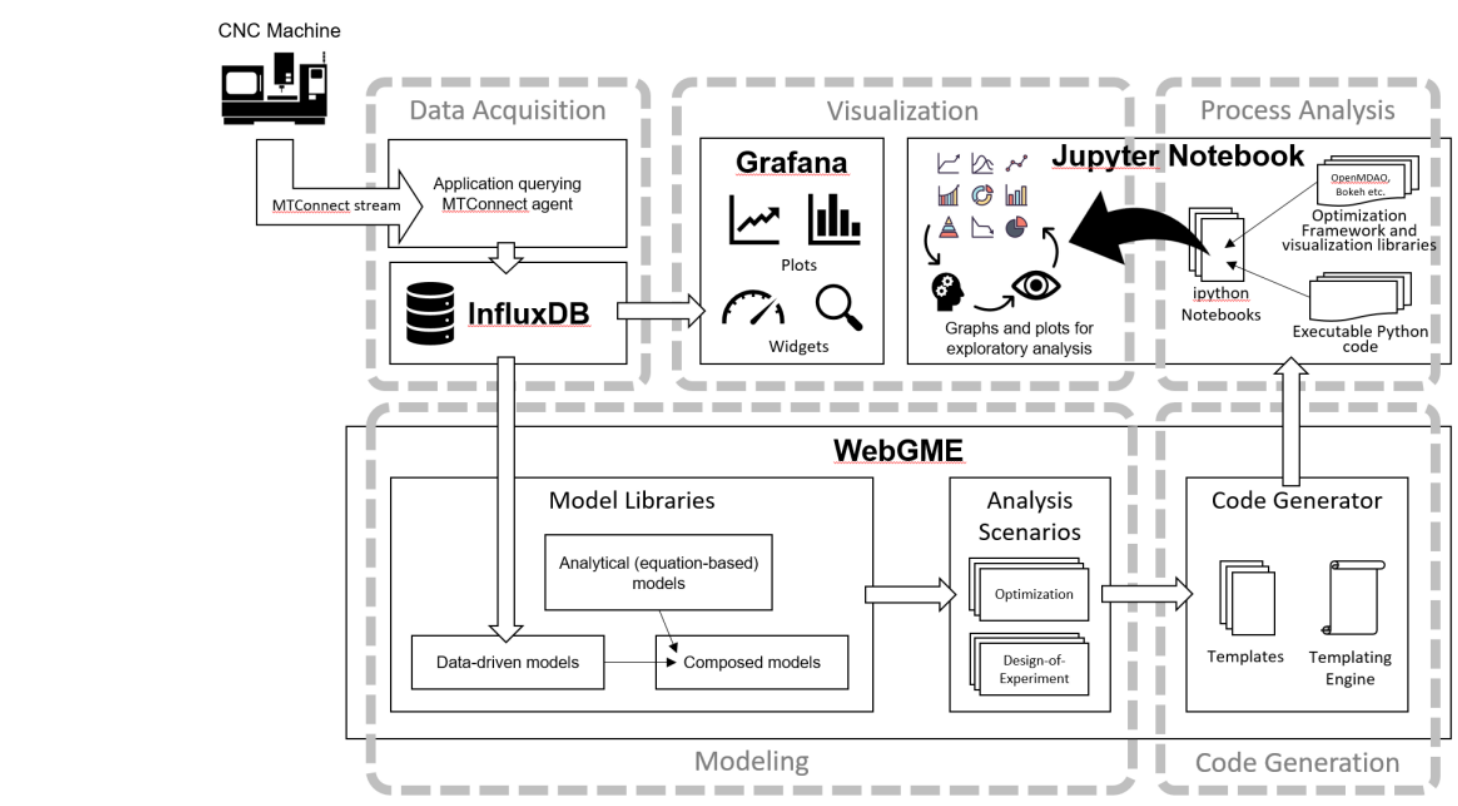

Figure 2. Detailed architecture of the framework

implementation of integrated tools, stepping towards the goal of being able to rapidly prototype ad-hoc analyses.

The analytical (i.e. equation-based) models as well as data-driven models of a production process can be used for various analyses like optimization, Design-ofExperiment (DoE), Discrete Event Systems (DES) simulations etc. The proposed framework uses MultiDisciplinary Analysis and Optimization (MDAO) methodology for specifying optimization and DoE analyses and DES simulations to provide long-term predictions of the system under study. Also, the framework uses regression techniques to approximate process models.

\section{Related work}

There have been efforts to design and implement an infrastructure to apply ad-hoc analyses to manufacturing processes. Tolio et al. describe such an integrated framework for the analysis and design of manufacturing systems [19]. Their virtual factory concept emphasizes the harmonization of heterogeneous information through a common data model, shared data storage and middleware to mediate the use of analytical tools. Relative to that work, we focus on efficiently assembling the tools to address unforeseen problems in processes and operations.

Using MTConnect and statistical models, Bengtsson et. al. determine the parameters to populate discrete event simulation (DES) models, which are used to analyze the underlying manufacturing process [20]. Shao et. al. use DES models [21] and Park \& Law et. al. use regression models [22], both of which are populated using MTConnect data. These examples show the benefits of MTConnect for data acquisition in the data-driven modeling paradigm. The work presented in this paper is complementary to the research mentioned above, by providing the tools to expedite the process of developing the predictive and analytical models.

\section{Framework}

In this section, we discuss the analytical framework in detail. The framework is an integrated environment for (1) acquiring process data, (2) developing models of the process using the acquired data, and (3) using the developed models to perform analyses. This integrated environment is built using WebGME [23], a web-based modeling platform, which provides the means to integrate all the software components needed to accomplish the tasks mentioned above. The integrated environment can be logically divided into three entities: (1) a domainspecific language, (2) an environment for code generation and execution, and (3) platforms for data acquisition and visualization. Figure 2 shows these software components in detail and the interfaces between them. Bold arrows in the figure signify the flow of data and artifacts between the individual components, and regular weight arrows signify the dependencies.

\subsection{The Domain-Specific Modeling Language}

Domain-Specific Modeling Languages (DSMLs) are modeling languages tailored for a particular domain, as opposed to generic modeling languages like UML [24]. The main benefit in using a DSML is that it exposes concepts that are already familiar to domain experts and can thus decrease the barrier to entry for the users who are knowledgeable in manufacturing but less experienced with data analysis. Additionally, a DSML provides the ability to compose models, to formally verify model compositions by enforcing constraints on the interfaces, and to improve the overall usability of the models with the help of code-synthesis [25].

Developing a DSML with WebGME begins with the definition of a meta-model, which expresses the concepts 
in the language as well as the relationships between those concepts. Figure 3 shows a grossly simplified version of the meta-model for our "MOdel Composition and Analysis" (MOCA) DSML.

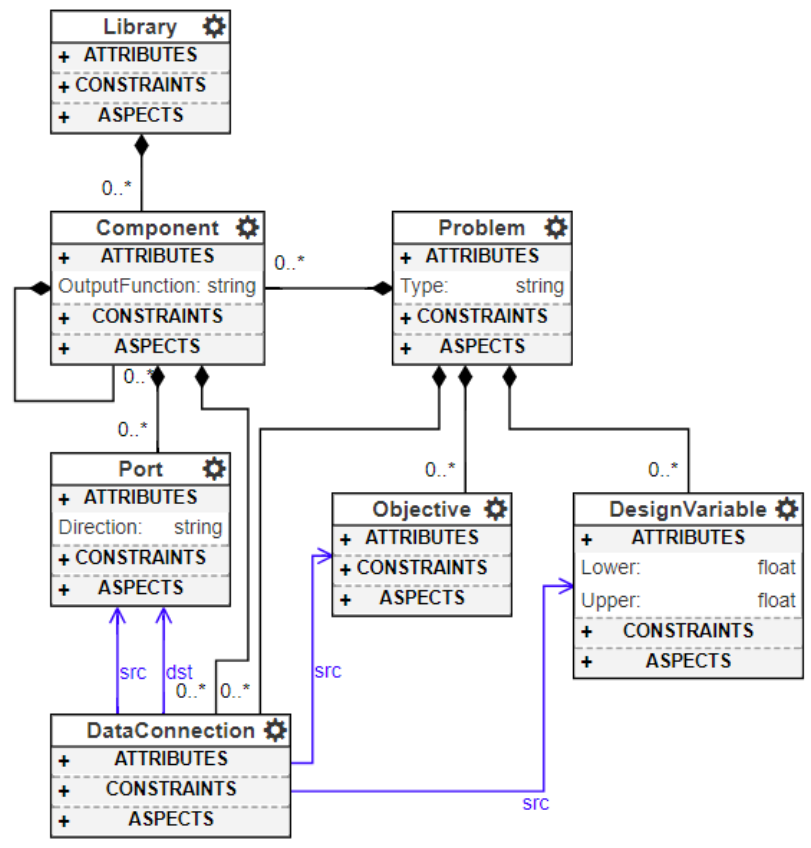

Figure 3. A simplified version of MOCA metamodel

The figure roughly translates to following set of rules: Library can contain one or more Components which can be reused, by creating Problem instances with different DesignVariables and Objectives. These Components can be composed to form assemblies of Components and the analysis such as Optimization or DoE can be specified over them using Problems.

Problems and Components provide separation of concern between defining the models and using them for analyses. Component specifies how the model will behave (i.e. its business logic). Problem specifies how the model (specified by an enclosed Component or an assembly of Components) will be used for the analysis, as it captures the information about the values to be assigned to the Ports of a Component (or an assembly of Components) during the optimization or a DoE analysis phase.

\subsubsection{Model definition}

The behavior of a manufacturing process is captured by a Component. For example, a user can create a Component named "TurningProcess" with input Ports "SpindleSpeed" and "FeedRate" and an output Port "ProductionTime". This Component itself contains the explicit mathematical relationship between its inputs and outputs, saved as an attribute "OutputFunction". The mathematical relationship is stored in the form of a function written in Python, which takes process parameters as inputs and returns the calculated production time. Since the Components can be composed, a complex analytical relation can be divided into several Components.
In case where the user does not have such a mathematical relationship, a data-driven approach can be used to deduce it using regression analyses. For this, the modeling language contains a concept called DataDrivenComponent. It can have Ports similar to a Component, but it contains an additional modeling concept called LearningAlgorithm which is used to learn the relationship between its inputs and outputs using data. The LearningAlgorithm specifies the type of regression analysis to be used, and also saves the learned models, so that they can be later used for analysis purposes, much like aforementioned Components. The regression algorithms are provided by Scikit-Learn, a machinelearning library in Python.

\subsubsection{Analysis specification}

Once the user has the model of the manufacturing process (derived either by data-driven techniques or defined explicitly), it can be used for various kinds of analyses by instantiating it within a Problem. A Problem can be set up in a way that it represents an Optimization analysis or a DoE analysis. In both the cases, A Problem specifies which Port(s) of the underlying enclosed Component are treated as design variables for that analysis scenario, by connecting them to DesignVariable(s). DesignVariables also provide the range in which the Ports are assigned values during the iterations of the optimizer or DoE driver routine. The optimization and DoE routines are part of OpenMDAO, which is an open-source, component-based library written in Python for MDAO analyses.

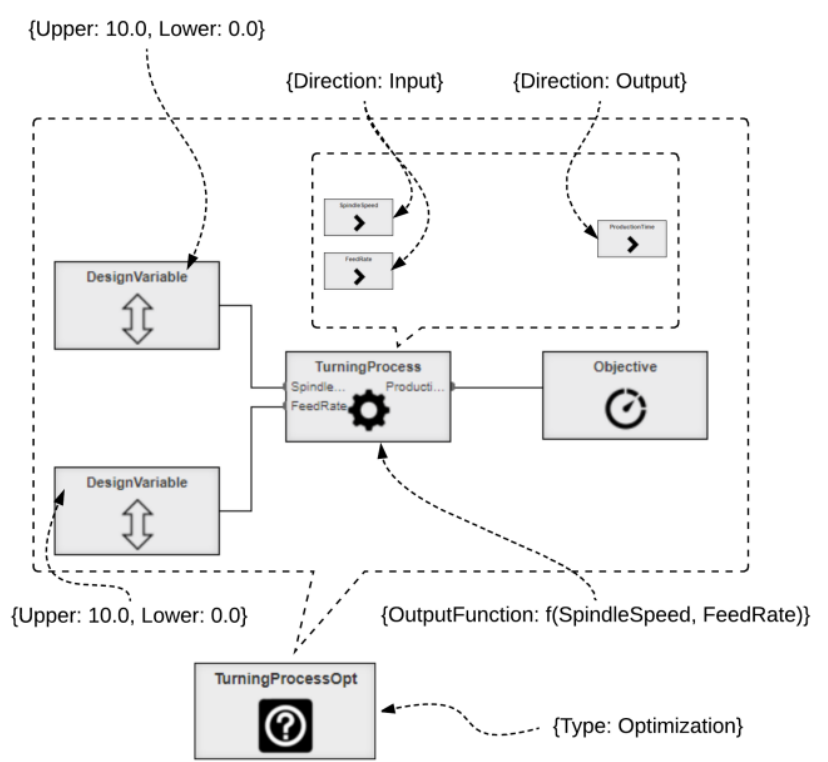

Figure 4. An example of a turning process model

In the example discussed above, the user may use a Problem (named "TurningProcessOpt" in the diagram) to specify an optimization scenario of the "TurningProcess", by associating "SpindleSpeed" and/or "FeedRate" inputs with DesignVariable(s) and "ProductionTime" output with Objective to be minimized. Figure 4 illustrates this by showing the hierarchy of these modeling entities along with their attributes. 


\subsection{Code generation and execution}

The actual analysis is performed by converting MOCA models into executable code. The web-based nature and client-server architecture of the WebGME tool means that the generated code can either reside on the serverside (where the WebGME tool is running as a service), or on the client-side (where the browser acts as a front-end using which the user interacts with the framework). Code generation is performed as follows - First, the code generator traverses the domain-specific model(s) and then populates the pre-defined code templates. The result is a fully executable Python codebase which uses the aforementioned libraries.

A Jupyter notebook is a web-based tool to publish readable and executable documents [26]. The generated Python code can be executed by using these Jupyter notebook documents, which are also generated. The generated Python code and Jupyter notebook documents can be saved and executed locally or remotely, the latter of which frees the user from the necessity of having the execution environment installed on their computer.

\subsection{Data acquisition and visualization}

One of the major strengths of this framework is its ability to integrate near real-time data-streams with the process models. Data acquisition consists of two phases: (1) querying an MTConnect agent to get the data from a CNC machine, and (2) storing the data in a database. Once the data is in the database, it can be queried during model training and/or model execution. To specify how data is stored, Database modeling concept can be used which captures the information about the URL of the MTConnect agent (which provides the stream of CNC data) and the unique URL to the database server where the data needs to be stored.

Visualizations provide insights into underlying physical phenomena. The visualization techniques used in the framework can be applied to both results of the analyses and raw MTConnect data stored in the database. For visualizing the results of the DoE and DES analyses, the generated code for the models uses a plotting library named Bokeh which can render scatter plots and surface plots in the Jupyter notebook environment. The code for visualizing the analyses is generated along with the code for models, which removes the burden of interacting with the plotting libraries from the user. The user can also visualize the raw MTConnect data using Grafana, which is a web-based tool for visualizing the contents of an InfluxDB database.

\section{Conclusions and future work}

This paper presents an analytical framework for smart manufacturing which allows users to perform ad-hoc analyses by creating domain-specific models that are used to specify input sources, analysis algorithms and visualizations. Code generation transforms these domainspecific models into the appropriate lower-level artifacts, such as Python code that uses a regression algorithm, optimizer and a visualizer, which helps manufacturing experts concentrate on analyses rather than low-level implementation details and tool integration. As discussed earlier, the web-service nature of the framework allows the user to run it on computationally restrained platforms. It also frees the user from additional overheads like installation of software components which are present for the offline counterparts of this tool. We believe that frameworks such as ours are key enablers of the smart manufacturing vision.

The future work includes the integration of the tool presented in the paper with the real-world high-fidelity machine data, which will allow the user to develop more detailed models of the processes. To make the tool more approachable for the industrial experts, it needs to be interoperable with other communication standards like MTConnect (e.g. OPC-UA [27]). The tool must be robust in the ways that it allows the models to be composed.

\section{References}

1. A. Vijayaraghavan, W. Sobel, A. Fox, D. Dornfeld, and P. Warndorf, "Improving machine tool interoperability using standardized interface protocols: MTconnect," Laboratory for Manufacturing and Sustainability (2008)

2. "Influxdb, version 1.3." [Online], https://www.influxdata.com/time-seriesplatform/influxdb (2017).

3. J. Gray, K. T. Moore, and B. A. Naylor, "Openmdao: An open source framework for multidisciplinary analysis and optimization," in AIAA/ISSMO Multidisciplinary Analysis Optimization Conference Proceedings, vol. 5 (2010).

4. N. Matloff, "Introduction to discrete-event simulation and the simpy language," Davis, CA. Dept of Computer Science. University of California at Davis. Retrieved on August, vol. 2, p. 2009 (2008).

5. F. Pedregosa, G. Varoquaux, A. Gramfort, V. Michel, B. Thirion, O. Grisel, M. Blondel, P. Prettenhofer, R. Weiss, V. Dubourg, J. Vanderplas, A. Passos, D. Cournapeau, M. Brucher, M. Perrot, and E. Duchesnay, "Scikit-learn: Machine learning in Python," Journal of Machine Learning Research, vol. 12, pp. 2825-2830 (2011).

6. Bokeh Development Team, Bokeh: Python library for interactive visualization (2014).

7. Grafana: the open platform for analytics and monitoring (2017).

8. S. Jeschke, C. Brecher, T. Meisen, D. O” zdemir, and T. Eschert, "Industrial internet of things and cyber manufacturing systems," in Industrial Internet of Things, pp. 3-19, Springer (2017).

9. J. Lee, E. Lapira, B. Bagheri, and H.-a. Kao, "Recent advances and trends in predictive manufacturing systems in big data environment," Manufacturing Letters, vol. 1, no. 1, pp. 38-41 (2013).

10. J. Lee, B. Bagheri, and H.-A. Kao, "A cyber-physical systems architecture for industry 4.0-based manufacturing systems," Manufacturing Letters, vol. 3, pp. 18-23 (2015) 
11. A. B. Feeney, S. Frechette, and V. Srinivasan, "Cyber-physical systems engineering for manufacturing," in Industrial Internet of Things, pp. 81-110, Springer (2017).

12. S. Friedenthal, R. Griego, and M. Sampson, "Incose model based systems engineering (mbse) initiative," in INCOSE 2007 Symposium (2007).

13. I. P. Arbizu and C. L. Perez, "Surface roughness prediction by factorial design of experiments in turning processes," Journal of Materials Processing Technology, vol. 143, pp. 390-396 (2003).

14. T. O" zel and Y. Karpat, "Predictive modeling of surface roughness and tool wear in hard turning using regression and neural networks," International Journal of Machine Tools and Manufacture, vol. 45, no. 4, pp. 467-479 (2005).

15. P. Suresh, P. V. Rao, and S. Deshmukh, "A genetic algorithmic approach for optimization of surface roughness prediction model," International Journal of Machine Tools and Manufacture, vol. 42, no. 6, pp. 675-680 (2002).

16. W. Li and S. Kara, "An empirical model for predicting energy consumption of manufacturing processes: a case of turning process," Proceedings of the Institution of Mechanical Engineers, Part B: Journal of Engineering Manufacture, vol. 225, no. 9, pp. 1636-1646 (2011).

17. P. Backus, M. Janakiram, S. Mowzoon, C. Runger, and A. Bhargava, "Factory cycle-time prediction with a data-mining approach," IEEE Transactions on Semiconductor Manufacturing, vol. 19, no. 2, pp. 252-258 (2006).

18. R. Bhinge, N. Biswas, D. Dornfeld, J. Park, K. H. Law, M. Helu, and S. Rachuri, "An intelligent machine monitoring system for energy prediction using a gaussian process regression," in 2014 IEEE International Conference on Big Data (Big Data), pp. 978-986, IEEE (2014).

19. T. Tolio, M. Sacco, W. Terkaj, and M. Urgo, "Virtual factory: An integrated framework for manufacturing systems design and analysis," Procedia CIRP, vol. 7, pp. 25-30 (2013).
20. N. Bengtsson, J. Michaloski, F. Proctor, G. Shao, and S. Venkatesh, "Towards data driven sustainable machining combining mtconnect production data and discrete event simulation," in Proceedings of the Proceedings of ASME 2010 International Manufacturing Science and Engineering Conference (2010).

21. G. Shao, S.-J. Shin, and S. Jain, "Data analytics using simulation for smart manufacturing," in Proceedings of the 2014 Winter Simulation Conference, pp. 2192-2203, IEEE Press (2014).

22. J. Park, K. H. Law, R. Bhinge, N. Biswas, A. Srinivasan, D. A. Dornfeld, M. Helu, and S. Rachuri, "A generalized data-driven energy prediction model with uncertainty for a milling machine tool using gaussian process," in ASME 2015 International Manufacturing Science and Engineering Conference, pp. V002T05A010-V002T05A010, American Society of Mechanical Engineers (2015).

23. M. Mar'oti, T. Kecsk'es, R. Keresk'enyi, B. Broll, P. V“olgyesi, L. Jur'acz, T. Levendovszky, and $A^{\prime}$. Le'deczi, "Next generation (meta) modeling: Weband cloud-based collaborative tool infrastructure.," MPM@MoDELS,vol. 1237,pp. 41-60 (2014).

24. J. Rumbaugh, I. Jacobson, and G. Booch, Unified modeling language reference manual, the. Pearson Higher Education (2004).

25. A'. Le'deczi, A. Bakay, M. Maroti, P. Volgyesi, G. Nordstrom, J. Sprinkle, and G. Karsai, "Composing domain-specific design environments," Computer, vol. 34, no. 11, pp. 44-51 (2001).

26. T. Kluyver, B. Ragan-Kelley, F. P'erez, B. E. Granger, M. Bussonnier, J. Frederic, K. Kelley, J. B. Hamrick, J. Grout, S. Corlay, et al., "Jupyter notebooks-a publishing format for reproducible computational workflows.," in ELPUB, pp. 87-90 (2016).

27. S.-H. Leitner and W. Mahnke, "Opc ua-serviceoriented architecture for industrial applications," ABB Corporate Research Center (2006). 